\title{
Multiparametric MRI for early detection of diffuse liver disease: an interview with David Breen
}

\author{
David J Breen*,1 \\ ${ }^{1}$ Consultant Abdominal Radiologist, Associate Professor of Radiology, University Hospital of Southampton, Southampton, UK \\ * Author for correspondence: david.breen@uhs.nhs.uk
}
"LiverMultiScan (LMS) is a noninvasive medical imaging software tool that can aid a clinician in diagnosing diffuse liver disorders”

David J Breen is Associate Professor of Radiology and previous Clinical Director of Radiology at University Hospital Southampton. His interests are in abdominal imaging and intervention, particularly of hepatopancreato-biliary (HPB)and renal disease. He has a major interest in image-guided tumor ablation and improving outcomes from these procedures. He has previously served as Vice Chair of the NHS England Clinical Reference Group in Interventional Radiology and for the NIHR/NCRN Upper GI Clinical Studies Group.

First draft submitted: 24 October 2017; Accepted for publication: 24 October 2017; Published online: 10 January 2018

Keywords: iron corrected T1 • liver disease • liver fibrosis • liver steatosis • magnetic resonance T1 mapping - magnetic resonance $\mathrm{T}^{\star}{ }^{\star} \bullet$ multiparametric MRI

\section{What is LiverMultiScan ${ }^{\mathrm{TM}}$ ?}

LiverMultiScan (LMS) is a noninvasive medical imaging software tool that can aid a clinician in diagnosing diffuse liver disorders. It uses multiparametric MRI without contrast agent, and is compatible with the majority of scanners. MRI is ideal for characterizing tissue, as it is sensitive to subtle differences in tissue composition, can sample the entire liver quickly, is safe, reproducible and yields objective measurements which can significantly contribute to prospective patient management. The LMS protocol takes less than $15 \mathrm{~min}$ to run and enables quantitative characterization of the liver, generating mapped metrics that correlate with inflammation/fibrosis, fat and iron. Importantly, LMS has both US FDA $(510[\mathrm{k}])$ clearance and CE marking, which means that it can be used safely in the USA and EU as an aid to diagnose patients with liver abnormalities.

\section{What imaging markers does the device measure?}

The LMS MRI protocol acquires transverse abdominal T1, T2* and proton density fat fraction maps to characterize liver tissue. T1 mapping is used in cardiology to assess fibroinflammatory disease in the myocardium; however, its application to the liver has been limited by the confounding effect of excess iron, a common occurrence in chronic liver disease, on T1 acquisition. LMS has overcome this barrier with proprietary technology to correct T1 for the effect of iron, which can be estimated from T2*. The corrected T1 (cT1) correlates well with liver-related outcomes in adults, such that a high cT1 is indicative of a greater degree of inflammation and fibrosis in the liver. The LMS protocol additionally measures liver fat with the proton density fat fraction. When combined, these imaging markers provide a fast, noninvasive and reproducible way to measure liver health.

\section{What are the advantages of using LiverMultiScan over liver biopsy?}

Liver biopsy has long been the gold standard for staging liver disease. However, it is painful, costly, carries a not insignificant risk of complications and allows sampling of only a small part of the liver $(1 / 50,000$ th $)$ that may not be representative of the rest of the liver. With liver disease reaching epidemic proportions in western populations,

Future $\because$ Medicine 
there is an urgent need for noninvasive technology that can identify patients with chronic liver disease, and stratify those at high risk of disease progression. Noninvasive imaging biomarkers, such as those measured with LMS, are attractive as they are safe, comfortable, examine whole slices of the liver and allow for serial/linear measurements to both characterize and monitor liver disease.

\section{How does LiverMultiScan compare with other noninvasive methods?}

Several other imaging-based methods have been developed to evaluate liver fibrosis. Noninvasive methods include ultrasound-based transient elastography and magnetic resonance elastography (MRE). Ultrasound-based elastography, such as the FibroScan ${ }^{\circledR}$ device (Echosens, France), measures shear wave velocity that gets passed into the liver from a small transducer attached to the end of an ultrasound probe. The advantage of ultrasound transient elastography is that it can be performed at the point-of-care, giving instantaneous results. However, it does not permit formal visualization and it can give unreliable readings if there is significant fat or fluid between the probe and the liver, such as in patients with obesity or ascites. MRE measures fibrosis by imaging the visco-elastic mechanical properties of the liver. With this technique, a passive mechanical driver is placed on the abdomen to generate shear waves at a defined frequency. These propagating waves travel faster in stiffer tissue than in softer tissue, and a magnetic resonance elastogram, or stiffness map, is generated. The advantage of MRE is that it can depict tissue stiffness over an entire liver cross-section, showing distribution over larger volumes. However, MRE requires additional expensive hardware, is compromised in patients with hemosiderosis, and lacks standardization across vendors.

The advantage of multiparametric MRI is that it measures several biomarkers of disease in a fast, reproducible way. It works equally well in obese patients and those with ascites or hemosiderosis. Furthermore, it is standardized across scanner manufacturers and magnet strengths, making it more widely available with increasing access to magnetic resonance scanners around the world, and gives more spatial information as it shows a map of the liver.

\section{Is LiverMultiScan cost-effective?}

Yes, in a recent modeling study assessing the cost-effectiveness of noninvasive techniques in diagnosing nonalcoholic fatty liver disease, LMS was shown to lead to significant cost savings for the National Health Service either as an adjunct to, or replacement for, transient elastography. In this study, three pathways were compared, using LMS alone, transient elastography alone or using LMS as an adjunct to transient elastography. The cost modeling showed that when LMS was used as an adjunct to transient elastography, it had the potential to reduce the number of liver biopsies by $66 \%$, and a reduction of $16 \%$ when LMS is used as a replacement to transient elastography.

\section{Does LiverMultiScan have any applications beyond chronic liver disease?}

Yes, the clinical utility of LMS is currently being evaluated in different clinical indications and in surgical planning for patients with cancer in the liver as part of a multicenter clinical study. It is also being evaluated in an ongoing prospective observational study to assess liver inflammation and fibrosis in a pediatric population.

\section{Disclaimer}

The opinions expressed in this article are those of the author and do not necessarily reflect the views of Future Medicine Ltd.

\section{Financial \& competing interests disclosure}

Research collaboration with the company producing LMS for scientific pursuits without any remuneration (nonfinancial relationship). The author has no other relevant affiliations or financial involvement with any organization or entity with a financial interest in or financial conflict with the subject matter or materials discussed in the manuscript apart from those disclosed.

No writing assistance was utilized in the production of this manuscript.

\section{Open access}

This work is licensed under the Attribution-NonCommercial-NoDerivatives 4.0 Unported License. To view a copy of this license, visit http://creativecommons.org/licenses/by-nc-nd/4.0/ 